\title{
Size DEPENDENCE OF A TEMPERATURE-INDUCED SOL- ID-SOLID PHASE TRANSITION IN COPPER(I) SULFIDE
}

\author{
Jessy B Rivest ${ }^{1,2}$, Lam-Kiu Fong ${ }^{3}$, Prashant K Jain ${ }^{2,3,4}$, Michael F Toney ${ }^{5}$, A Paul Alivisatos ${ }^{2,3 *}$ \\ 1 Mechanical Engineering Department, UC Berkeley. Berkeley, CA 94720, United States \\ 2 Materials Science Division, Lawrence Berkeley National Laboratory. Berkeley, CA 94720, United States \\ 3 Department of Chemistry, UC Berkeley. Berkeley, CA 94720, United States \\ 4 Miller Institute for Basic Research in Science, UC Berkeley. Berkeley, CA 94720, United States \\ 5 SLAC National Accelerator Laboratory, Menlo Park, CA 94025, United States
}

KEYWORDS phase transition, low high chalcocite, nanoparticle, copper sulfide

CORRESPONDING AUTHOR

*ALivis@Berkeley.edu

\begin{abstract}
Determination of the phase diagrams for the nanocrystalline forms of materials is crucial for our understanding of nanostructures and the design of functional materials using nanoscale building blocks. The ability to study such transformations in nanomaterials with controlled shape offers further insight into transition mechanisms and the influence of particular facets. Here we present an investigation of the size-dependent, temperature-induced solid-solid phase transition in copper sulfide nanorods from low- to high-chalcocite. We find the transition temperature to be substantially reduced, with the high chalcocite phase appearing in the smallest nanocrystals at temperatures so low that they are typical of photovoltaic operation. Size dependence in phase transformations suggests the possibility of accessing morphologies that are not found in bulk solids at ambient conditions. These otherwise-inaccessible crystal phases could enable higher-performing materials in a range of applications, including sensing, switching, lighting, and photovoltaics.
\end{abstract}

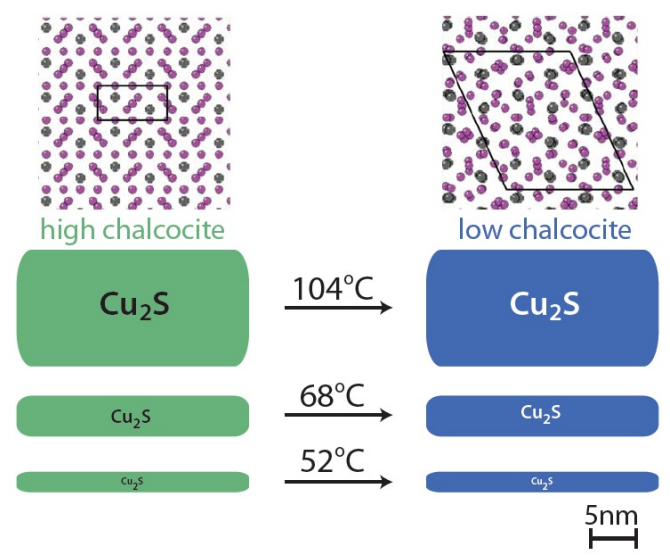


Size dependent structural transformations and phase transitions in nanoscale materials play a key role in determining the phases that can be accessed and employed at the nanoscale, and yet there are still only a limited number of such transformations whose size and shape dependence has been mapped out. As a result, our understanding of the underlying factors that control metastabilty and stability in nanocrystals is still incomplete. With the advent of the capability to control the shapes of nanocrystals, as well as the ability to separately tune composition through means such as ion exchange, it is now possible to investigate size dependent structural transformations in a much wider range of systems. Here we investigate one such structural transformation in nanorods of copper sulfide.

Over the past few decades, copper sulfide has captured the attention of a broad scientific community with a rich $\mathrm{Cu}_{2-x} \mathrm{~S}$ phase diagram and compositionally tunable properties. This semiconductor has found applications in photovoltaics, ${ }^{1}$ switching 2,3 and sensing ${ }^{4,5}$ (sometimes enabled or enhanced by chemical transformations ${ }^{6,7}$ or doping $\left.{ }^{8,9}\right)$, in addition to copper ore processing. ${ }^{10}$ The recent synthesis of copper(I) sulfide nanocrystals, $611-14$ may enable inexpensive solution processing for many of these applications, however, it is critical for implementation that the size-dependence of the material properties and crystallographic phase diagram be well understood.

Buffat and Borel described the size dependence of melting transitions in $1976^{15}$ and their work has been followed by a variety of experiments probing size dependence in temperature- or pressure-induced solidsolid and solid-liquid phase transitions. ${ }^{16-19}$ This body of work has described situations where thermodynamic factors dominate over kinetics, and in the simplest case, the size dependence arises from a competition between surface energy and internal energy of each crystallographic phase. As the surface area to volume ratio increases, phases with lower surface energy become more favorable:

$$
\begin{array}{ll}
T_{b}-T_{m}=\frac{2 T_{m}}{\Delta H^{0} \rho_{1} R}\left[\gamma_{1}-\gamma_{2}\left(\frac{\rho_{1}}{\rho_{2}}\right)^{2 / 3}\right], 17 & \text { Eqn } 1 \\
P_{b}-P_{m}=\frac{2}{R}\left(\gamma_{1}-\gamma_{2}\right),{ }^{20} & \text { Eqn } 2
\end{array}
$$

where $T_{b}\left(P_{b}\right)$ is the bulk transition temperature (pressure), $T_{m}\left(P_{m}\right)$ is the size-modified phase transition temperature (pressure), $\Delta \mathrm{H}^{\mathrm{o}}$ is the enthalpy change for the phase transition, $\mathrm{R}$ is the particle radius, $\rho$ is density, and $\gamma$ is the surface tension of each phase (1 or 2).

Depending on the nature of the phase transition, a reduction in nanoparticle size may either increase or decrease the temperature or pressure at which the transition occurs. In the case of a solid-liquid transition, the surface energy almost uniformly decreases, so a melting point depression is typically observed with reduced particle size (exceptions arise in cases of melting of inclusions of one solid phase encapsulated inside anoth- 
er solid). Solid-solid phase transitions have different trends: they can show either higher or lower transition temperature or pressure with decreasing particle size. While there have been many reports of pressureinduced solid-solid phase transitions, temperature-induced first-order solid-solid transitions have been difficult to reliably observe due to inadvertent particle reorganization, sublimation, or sintering, which can change the size dispersion.

Stoichiometric or nearly stoichiometric $\mathrm{Cu}_{2} \mathrm{~S}$ exhibits the monoclinic low-chalcocite phase at room temperature, and shows a bulk transition to a higher symmetry hexagonal high-chalcocite at $376.65 \mathrm{~K},{ }^{21} \mathrm{a}$ temperature low enough to avoid sintering. Low chalcocite is a p-type semiconductor self-doped by copper vacancies, and has relatively high carrier mobility. ${ }^{22,23}$ In the low chalcocite form, the sulfur sublattice is nearly close-packed hexagonal, while the copper ions are organized in a complex fashion, forming a 144-atom unit cell, $\mathrm{Cu}_{96} \mathrm{~S}_{48} \cdot{ }^{24}$ This arrangement of cations leads to an indirect bandgap ${ }^{25}$ of $\sim 1.2 \mathrm{eV}$. As low chalcocite (LC) converts topotactically to high chalcocite (HC), the sulfur atoms approximately maintain their positions while the copper ions adopt a partly statistical distribution on lattice sites, and the distinction between interstitial and lattice ions is lost. ${ }^{26}$ This 'melting' of the copper sublattice gives rise to an increased almost liquid-like ionic mobility, but a decreased hole conductivity. It is interesting to note that disorder in the crystal structure in the HC phase leads to reduced mobility for holes and electrons in what amounts to the higher symmetry phase. ${ }^{26}$ The transition has a subtle effect on the indirect bandgap (increased to $1.42 \mathrm{eV}$ ), but leaves the direct transitions unaffected. ${ }^{25}$ Despite the dependence of device-relevant optoelectronic properties on the copper(I) sulfide phase, there is lack of robust experimental data on phase properties of copper(I) sulfide, as it is nontrivial to identify its low-symmetry phases and to measure the exact stoichiometry, which varies between $\mathrm{x}=0$ and $\mathrm{x}=0.2$.

Phase transitions in copper(I) sulfide have been studied in extended solids ${ }^{27-29}$ and, more recently, in nanoparticles. ${ }^{30,31}$ A recent study ${ }^{32}$ illuminated the dynamics of the LC-HC phase transition in nanorods, enabling the visualization of fluctuations between these two phases during the nucleation and growth of HC from the LC phase. This study was performed without intentional heating, yet was able to access the HC phase likely due to beam heating. There have been additional reports of $\mathrm{HC}$ nanoparticles existing at low temperatures, ${ }^{11,13}$ which suggests a size dependence to copper(I) sulfide phase stability. However, until now, the nature of the relationship between particle size and transition temperature has not been systematically studied for the important phase transition from low to high chalcocite. Here, we show such a study using copper(I) sulfide nanorods to map out a size-dependent phase diagram for use in nanostructured devices employing nearly stoichiometric $\mathrm{Cu}_{2} \mathrm{~S}$ phases. 


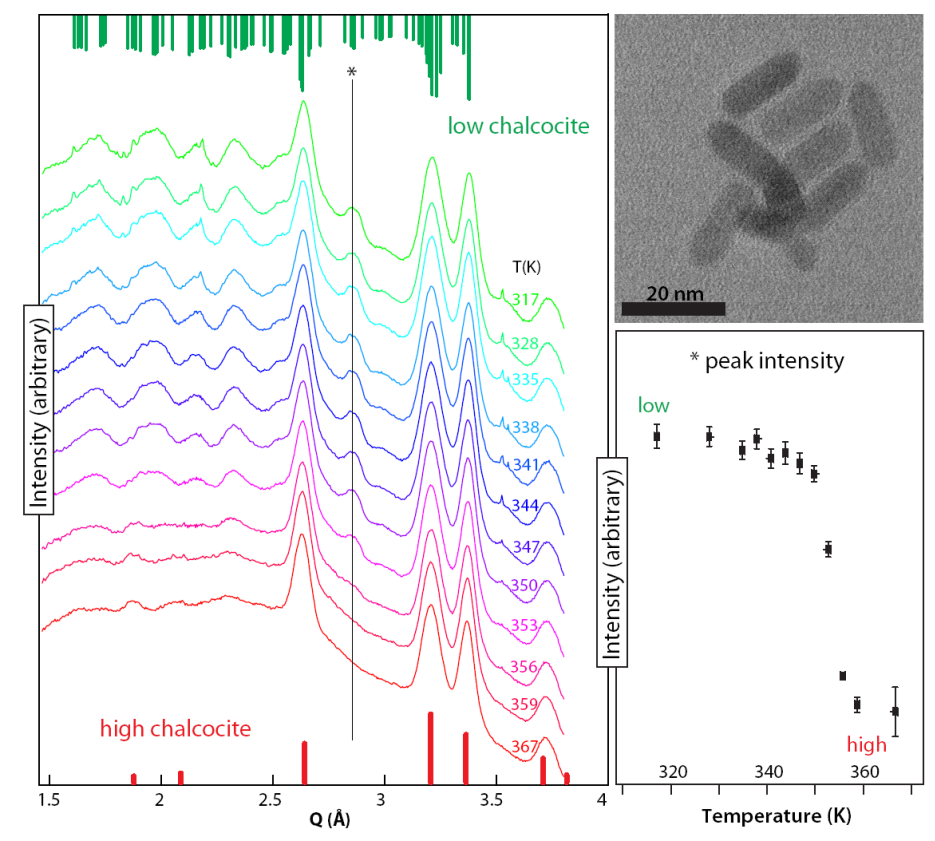

Figure 1. (left) Phase transition of $\mathrm{Cu}_{2} \mathrm{~S}$ from low chalcocite [Joint Committee on Powder Diffraction Standards (JCPDS) no. 00-0330490 ] to high chalcocite [JCPDS no. 00-046-1195] with increasing temperature. The * peak near $2.84 \AA$ is unique to low chalcocite, while other strong peaks are shared by the high chalcocite phase or are obscured by small crystallite size-induced broadening or background. The curves are offset for clarity. (top right) Transmission electron microscopy image of the nanorod sample from this data set allows size distribution measurements. (bottom right) A plot of the * peak intensity versus temperature shows a sharp transition from low to high chalcocite.

The stoichiometric low chalcocite phase of $\mathrm{Cu}_{2} \mathrm{~S}$ can be reliably obtained through a cation exchange of cadmium sulfide with $\mathrm{Cu}(\mathrm{I}) .9,21,33$ Monodisperse samples of $\mathrm{CdS}$ nanorods of controlled diameter and length were synthesized using Schlenck line techniques ${ }^{34}$, and then converted to $\mathrm{Cu}_{2} \mathrm{~S}$ by addition of a methanolic solution of copper(I) salt to a solution of CdS nanorods in toluene. ${ }^{6}$ The resulting copper(I) sulfide nanoparticles were drop-cast into a $\sim 500 \mathrm{~nm}$ thick film on a $\mathrm{Si}_{3} \mathrm{~N}_{4}$ substrate. All steps were performed air-free to prevent oxidation of the nanoparticles, which is known to result in a variation in the stoichiometry and phase of $\mathrm{Cu}_{2} \mathrm{~S} .{ }^{9} \mathrm{X}$-ray diffraction of the copper(I) sulfide films was measured at the Stanford Synchrotron Radiation Lightsource (SSRL) at beamline 11-3 with an energy of $12.735 \mathrm{keV}$ and a grazing incidence geometry. Samples were placed on a heating stage in a helium chamber and heated at 1 Kelvin per minute (to avoid temperature overshoot and facilitate near-equilibrium measurements). Collection time was 5 min and raw data were processed using WxDiff35 to obtain intensity (I) versus $\mathrm{Q}$ plots.

From a plot of $\mathrm{I}(\mathrm{Q})$, the strongest LC peak was identified and monitored as a function of temperature. Due to the crystallographic relationship between low and high chalcocite, almost all diffraction peaks from HC are shared with LC, but the greater and more complex ordering in the LC phase is manifested as several additional peaks. One such peak near $2.84 \AA$ is unique to LC and is denoted by * in Figure 1, which shows the temperature dependent diffraction. High chalcocite data closely matched the JCPDS standard 00-046-1195, 
while low chalcocite closely matched 00-033-0490 both before heating and after cooling, indicating the $\mathrm{Cu}_{2} \mathrm{~S}$ stoichiometry was maintained during transition.

Error bars on the data result from sample size distribution, as measured by transmission electron microscopy (TEM) (x-axis), and limits in the accuracy of a) temperature measurement, b) Gaussian fitting and diffraction peak integration and c) the Boltzmann fitting (y-axis). Errors in absolute TEM size measurements are assumed to be negligible in comparison with other errors mentioned above. As the particle size decreases, the diffraction peaks are broadened, introducing more uncertainty. This accounts for the general trend of larger error bars on smaller particles.

The integrated peak intensity $\left(\mathrm{A}_{\text {low chalcocite }}=\mathrm{A}_{\mathrm{LC}}\right)$ for the low chalcocite peak $(2.84 \AA)$ was plotted as a function of temperature, resulting in a sigmoidal curve representing the transition from low chalcocite $\left(\mathrm{A}_{\mathrm{LC}}>0\right)$ to high chalcocite $\left(\mathrm{A}_{\mathrm{HC}} \sim 0\right)$. Fitting these data with a Boltzmann function (Eqn 3) yielded a phase transition temperature, $\mathrm{T}_{0}$.

$$
y=\frac{A_{L C}-A_{H C}}{1+e^{\left(T-T_{o}\right) / \Delta T}}, \quad \text { Eqn } 3
$$

where $\Delta \mathrm{T}$ is the temperature range in which the transition occurs.

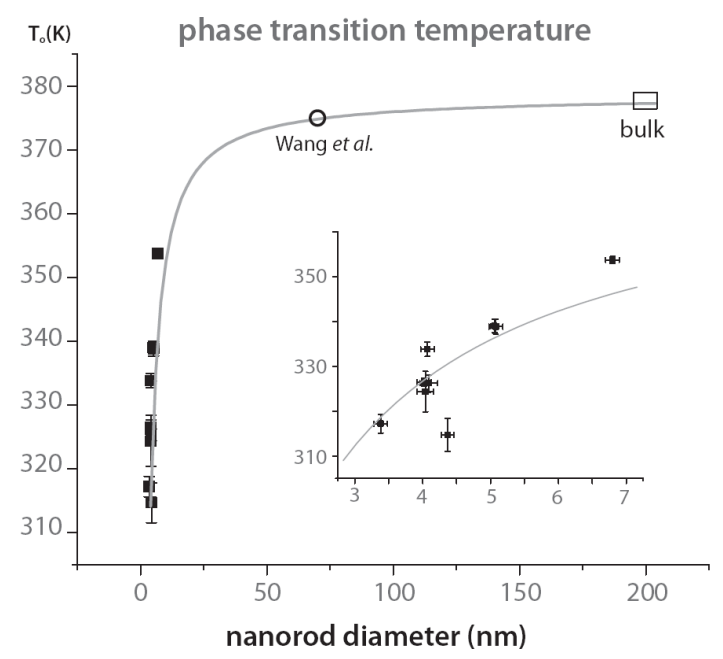

Figure 2. Phase transition temperature (Kelvin) as a function of nanorod diameter (nm). Data points collected in this study are black squares; data from bulk ${ }^{21}$ and from Wang et al.31 are noted. Inset shows a magnified plot of the data from the present study. The fitting curve (same in both plots) follows Equation 1 (Pearsons's $\chi^{2}=1.18$ ).

The average transition temperature $\left(\mathrm{T}_{0}\right)$ was plotted versus nanorod size (diameter) to obtain the size dependence (see Figure 2). A strong size dependence was observed, with nanorods transforming to high chalcocite at temperatures as low as $315 \mathrm{~K}$, a depression of more than $60 \mathrm{~K}$ below the bulk transition temperature of $376.65 \mathrm{~K}$. This trend indicates that $\mathrm{HC}$ may be thermodynamically favored at room temperature in nanocrystals smaller than $2.5 \mathrm{~nm}$ diameter. The data were fitted to Equation 1 by assuming the same density $\left(5.6 \mathrm{~g} / \mathrm{cc}^{24}\right)$ for the two phases, and an enthalpy change of $3849.3 \mathrm{~J} / \mathrm{mol} .{ }^{36}$ This yields a change in average 
surface energy of $20 \mathrm{mN} / \mathrm{m}$ or roughly a change of $50 \%$ from the low chalcocite value of $\sim 40 \mathrm{mN} / \mathrm{m} .{ }^{37,38}$ A typical range found for $\Delta \gamma / \gamma$ is $15 \%$ to $50 \% .^{19,39-41} \mathrm{Cu}_{2} \mathrm{~S}$ may be on the high end of this range due to the unique nature of its LC-HC transition in which the $\mathrm{Cu}$ sublattice effectively melts, lending some solid-liquid character to this solid-solid transition. Further demonstrating the LC-HC order-disorder character is the high formation entropy of $10.238 \mathrm{~J} / \mathrm{K}-\mathrm{mol}$ (with $1.3 \mathrm{Cu}(\mathrm{I})$ atoms distributed over 2 equivalent positions and 2.7 $\mathrm{Cu}(\mathrm{II})$ atoms distributed over 12 positions ${ }^{42}$ ).

The surface tensions obtained can be used in the Dupré equation to estimate the interfacial energy between the two phases (assuming no work of adhesion). Comparing interfacial energy with internal formation energy or Gibbs free energy $\Delta \mathrm{G}$, we can calculate the critical nucleation radius ( $\mathrm{R}^{*}$ ) to be $0.885 \mathrm{~nm}$, which is comparable to the smallest nuclei that were observed in the recent in situ TEM study of the this $\operatorname{transition}^{32}$ :

$$
R^{*}=\frac{2\left(\gamma_{L C}-\gamma_{H C}\right)}{\Delta G} \quad \cdot{ }^{43} \quad \text { Eqn } 4
$$

We also note that the hysteresis observed in this experiment (see Supporting Information) is similar to that observed in extended solids. ${ }^{27}$

$\mathrm{T}_{\mathrm{o}}$ was plotted as a function of nanorod length (keeping diameter constant at $4 \mathrm{~nm}$ ) and also as a function of nanorod diameter (keeping length constant at 40nm). We see that the nanorod transition temperature depends sensitively on the nanorod diameter $d$, whereas the nanorod length $l$ has no significant effect on $\mathrm{T}_{\mathrm{o}}$ (Figure 3 ). This is consistent with the fact that the independent variable in the size-dependent depression is the surface-area-to-volume ratio of the nanorods given by $(4 / d+1 / \ell)$. In the range of dimensions in the present study, the length term $(\ell)$ is negligible. 

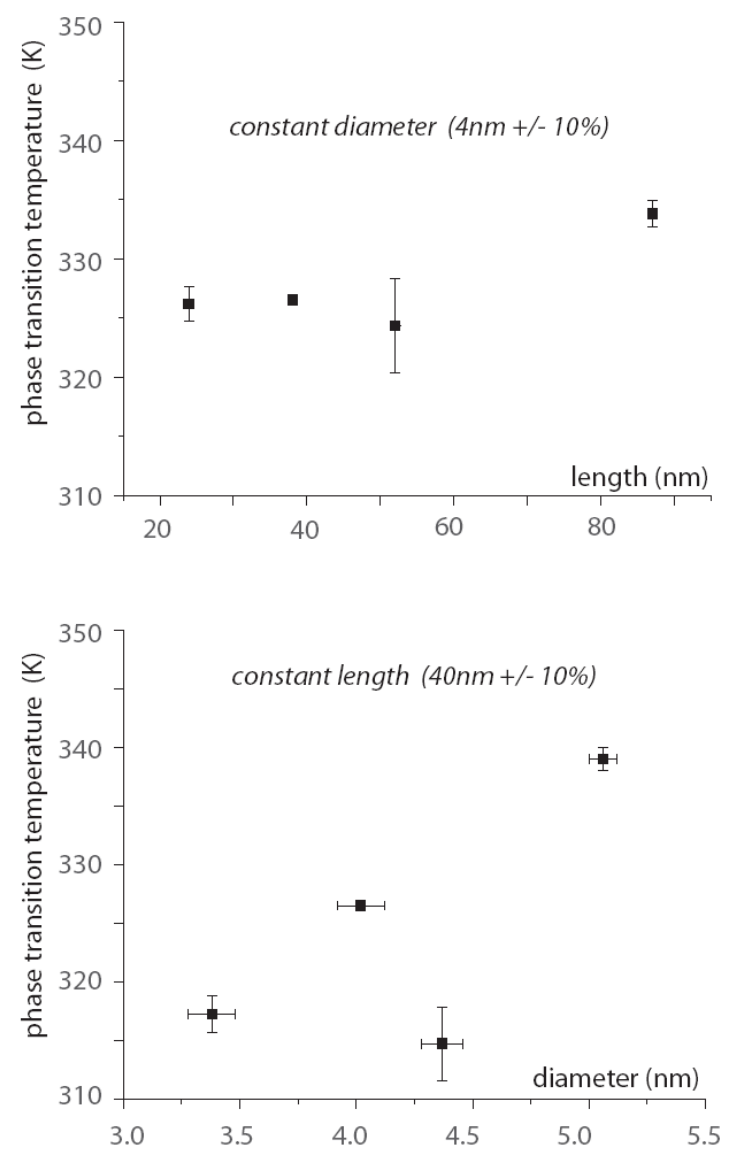

Figure 3. Phase transition temperatures (top) as a function of nanorod length with constant diameter of $4 \mathrm{~nm}$ ) and (bottom) as a function of nanorod diameter (with constant length of $40 \mathrm{~nm}$ ).

This work has some implications for the field of photovoltaics; copper sulfide comprised the original thin film solar cell in $1954 .^{33,44}$ In the photovoltaics community there has recently been significant interest in nanocrystalline $\mathrm{Cu}_{2} \mathrm{~S}$ as well as the related phases copper (indium gallium) (sulfide selenide) (CIGS) and copper zinc tin sulfide (CZTS) ${ }^{45,46}$, which employ an ordered cation substitution for improved stability and optical tuning. The phase diagrams of these ternary and quaternary semiconductors are also complex, leaving open the possibility of relatively low-temperature phase transitions that may show a strong sizedependence near photovoltaic operating temperatures (typically $300 \mathrm{~K}$ to $330 \mathrm{~K}$ ) or during crystal growth $(500-800 \mathrm{~K})$. In the case of $\mathrm{Cu}_{2} \mathrm{~S}$ for photovoltaic operation, our work indicates that it is crucial to maintain a particle size $>6 \mathrm{~nm}$ in diameter to avoid the stabilization of a deleterious phase (high chalcocite) at typical operating temperatures.

The stabilization of a high-temperature crystallographic phase at room temperature by use of nanocrystalline semiconductors may have favorable or unfavorable consequences, depending on the functional properties of interest. For example, the low to high ionic conductivity change during the LC-HC transition could find use in switching applications, which may be enabled by the narrow range of metastability observed due 
to the $\sim 10 \mathrm{~K}$ hysteresis of this transition. Our results also provide context for several reported observations of high chalcocite phases in nanoparticles existing at low temperatures. ${ }^{11,13}$ The uniquely low-temperature solid-solid transition observed for copper(I) sulfide suggests real-world applicability of this phase-change material, while making a broader call for characterization of additional materials whose phase diagrams enable transitions between functional crystal phases at room temperature.

\section{ACKNOWLEDGMENT}

We gratefully acknowledge Haimei Zheng for helpful discussions and Bryce Sadtler for providing some samples and for review of the manuscript. This work was supported by the Physical Chemistry of Semiconductor Nanocrystals Program, KC3105, Director, Office of Science, Office of Basic Energy Sciences, of the United States Department of Energy under contract DE-AC02-05CH11231. Portions of this research were carried out at the Stanford Synchrotron Radiation Lightsource, a national user facility operated by Stanford University on behalf of the U.S. Department of Energy, Office of Basic Energy Sciences. JBR received funding from an Intel Fellowship. PJ acknowledges the fellowship of the Miller Institute at UCB.

Supporting Information Available: Hysteresis data and data collection details. This material is available free of charge via the Internet at http://pubs.acs.org.

\section{REFERENCES}

(1) Hadley, H. Materials aspects of Cu2S for CdS/Cu2S solar cells. Journal of Crystal Growth 1977, 39, 61-72.

(2) Sakamoto, T.; Sunamura, H.; Kawaura, H.; Hasegawa, T.; Nakayama, T.; Aono, M. Nanometer-scale switches using copper sulfide. Appl. Phys. Lett. 2003, 82, 3032-3034.

(3) You, L. Copper Sulfide Solid-State Electrolytic Memory Devices, Case Western Reserve University Thesis, 2007.

(4) Sagade, A. Copper sulphide $\left(\mathrm{Cu}_{\mathrm{x}} \mathrm{S}\right)$ as an ammonia gas sensor working at room temperature. Sensors and Actuators B: Chemical 2008, 133, 135-143.

(5) Ames, M.; Liu, C.; Connor, S.; Horne, R.; Li, K.; Cui, Y. Smart Nanosensors for In-Situ Temperature Measurement in Fractured Geothermal Reservoirs. Proceedings, Thirty-Sixth Workshop on Geothermal Reservoir Engineering 2011.

(6) Sadtler, B.; Demchenko, D. O.; Zheng, H.; Hughes, S. M.; Merkle, M. G.; Dahmen, U.; Wang, L.-W.; Alivisatos, A. P. Selective facet reactivity during cation exchange in cadmium sulfide nanorods. J. Am. Chem. Soc. 2009, 131, 5285-5293.

(7) Luther, J. M.; Zheng, H.; Sadtler, B.; Alivisatos, A. P. Synthesis of PbS nanorods and other ionic nanocrystals of complex morphology by sequential cation exchange reactions. J. Am. Chem. Soc. 2009, 131, 16851-16857.

(8) Zhao, Y.; Pan, H.; Lou, Y.; Qiu, X.; Zhu, J.; Burda, C. Plasmonic Cu(2-x)S nanocrystals: optical and structural properties of copper-deficient copper(I) sulfides. J. Am. Chem. Soc. 2009, 131, 4253-4261.

(9) Luther, J. M.; Jain, P. K.; Ewers, T.; Alivisatos, A. P. Localized surface plasmon resonances arising from free carriers in doped quantum dots. Nat Mater 2011, 10,361-366.

(10) Dannenberg, R. O.; Gardner, P.C.; Crane, S. R.; Seidel, D. C. Recovery of cobalt and copper from complex sulfide concentrates; US Department of the Interior,Pittsburgh, PA, 1987.

(11) Sigman, M. B.; Ghezelbash, A.; Hanrath, T.; Saunders, A. E.; Lee, F.; Korgel, B. A. Solventless Synthesis of Monodisperse Cu 2S Nanorods, Nanodisks, and Nanoplatelets. J. Am. Chem. Soc. 2003, 125, 16050-16057.

(12) Wu, Y.; Wadia, C.; Ma, W.; Sadtler, B.; Alivisatos, A. P. Synthesis and photovoltaic application of copper(I) sulfide nanocrystals. Nano Lett. 2008, 8, 2551-2555.

(13) Liu, Z.; Xu, D.; Liang, J.; Shen, J.; Zhang, S.; Qian, Y. Growth of Cu2S ultrathin nanowires in a binary surfactant solvent. J Phys Chem B 2005, 109, 10699-10704.

(14) Rivest, J. B.; Swisher, S. L.; Fong, L.-K.; Zheng, H.; Alivisatos, A. P. Assembled monolayer nanorod heterojunctions. ACS Nano 2011, 5, 3811-3816.

(15) Buffat, P.; Borel, J.-P. Size effect on the melting temperature of gold particles. Phys. Rev. A 1976, 13, $2287-2298$.

(16) Tolbert, S. H.; Alivisatos, A. P. Size Dependence of a First Order Solid-Solid Phase Transition: The Wurtzite to Rock Salt Transformation in CdSe Nanocrystals. Science 1994, 265, 373-376.

(17) Goldstein, A. N.; Echer, C. M.; Alivisatos, A. P. Melting in semiconductor nanocrystals. Science 1992, 256, $1425-1427$.

(18) McHale, J. M. Surface Energies and Thermodynamic Phase Stability in Nanocrystalline Aluminas. Science 1997, 277, 788-791.

(19) Banfield, J. Particle size effects on transformation kinetics and phase stability in nanocrystalline TiO2. American Mineralogist 1997, 82, 717-728.

(20) Tolbert, S. H.; Alivisatos, A. P. High-Pressure Structural Transformations in Semiconductor Nanocrystals. Annu. Rev. Phys. 
Chem. 1995, 46, 595-626.

(21) Sands, T. D.; Washburn, J.; Gronsky, R. High Resolution Observations of Copper Vacancy Ordering in Chalcocite (Cu2S) and the Transformation to Djurleite (Cu1.97 to 1.94S). Phys. Stat. Sol. (a) 1982, 72, 551-559.

(22) Hirahara, E. The Electrical Conductivity and Isothermal Hall Effect in Cuprous Sulfide, Semi-Conductor. Journal of the Physical Society of Japan 1951, 6, 428-437.

Bougnot, J.; Guastavino, F.; Luquet, H.; Sodini, D. On the electrical properties of CuxS. Phys. Stat. Sol. (a) 1971, 8, K93-K96. Buerger, M. J.; Buerger, N. W. Low-Chalcocite and High-Chalcocite. The American Minerologist 1944, $29,55-65$. Rastogi, A.; Salkalachen, S. Optical absorption behaviour of evaporated CuxS thin films. Thin Solid Films 1982, 97, 191-199. Okamoto, K.; Kawai, S. Electrical Conduction and Phase Transition of Copper Sulfides. Jpn. J. Appl. Phys. 1973, 12, 11301138 . Leon, M.; Terao, N. On the phase transition of chalcocite Cu2S thin films. Phys. Stat. Sol. (a) 1981, 67, K11-K14. Putnis, A. Electron diffraction study of phase transformations in copper sulfides. American Mineralogist 1977, 62, $107-114$. Kazinets, M. M.; Ivanova, I. V.; Shafizade, R. B. Investigation of phase transformations in Cu1.8S-Cu2S thin films by kinematic electron diffraction. GPHT 1979, 1, 199-206. Lotfipour, M.; Machani, T.; Rossi, D. P.; Plass, K. E. $\alpha$-Chalcocite Nanoparticle Synthesis and Stability. Chem. Mater. 2011, in press.

Wang, S. Phase transitions in the $\mathrm{Cu} 2 \mathrm{~S}$ nanowires. Materials Chemistry and Physics 2002, 75, 32-38.

Zheng, H.; Rivest, J. B.; Miller, T. A.; Sadtler, B.; Lindenberg, A.; Toney, M. F.; Wang, L. W.; Kisielowski, C.; Alivisatos, A. P. Observation of Transient Structural-Transformation Dynamics in a Cu2S Nanorod. Science 2011, 333, $206-209$. Carlson, A. Research in semiconductor films; 97494th ed. WADC Technical Report, 1956; Vol. Clevite Corporation. Peng, Z. A.; Peng, X. Formation of High-Quality CdTe, CdSe, and CdS Nanocrystals Using CdO as Precursor. J. Am. Chem. Soc. 2001, 123, 183-184.

Mannsfeld, S. C. B.; Virkar, A.; Reese, C.; Toney, M. F.; Bao, Z. Precise Structure of Pentacene Monolayers on Amorphous

Silicon Oxide and Relation to Charge Transport. Adv. Mater. 2009, 21, 2294-2298.
Thermodynamic Properties of Compounds, CuS to ErF3, pp. 175-200; Scientific Group Thermodata Europe SGTE, E. T. H. R. A., Ed. 2001; pp. 1-26.

Ozcan, O. Classification of minerals according to their critical surface tension of wetting values. International Journal of Mineral Processing 1992, 34, 191-204.

Janczuk, B.; Wójcik, W .; Zdziennicka, A. Determination of surface-free energy components of synthetic chalcocite from contact angle measurements. Powder Technology 1993, 76, 233-239.

Tolbert, S. H.; Alivisatos, A. P. Size Dependence of a First Order Solid-Solid Phase Transition: The Wurtzite to Rock Salt Transformation in CdSe Nanocrystals. Science 1994, 265, 373-376.

(41) Hardy, S. C.; Coriell, S. R. Surface tension and interface kinetics of ice crystals freezing and melting in sodium chloride solutions. Journal of Crystal Growth 1973, 20, 292-300.

(42) Gronvold, F.; Westrum, E. Thermodynamics of copper sulfides I. Heat capacity and thermodynamic properties of copper (I) sulfide, Cu2S, from 5 to $950 \mathrm{~K}^{*} 1$. The Journal of Chemical Thermodynamics 1987, 19, 1183-1198.

(43) Schmelzer, J.; Röpke, G. Nucleation theory and applications; 2005; pp. 77-79. Reynolds, D.; Leies, G.; Antes, L.; Marburger, R. Photovoltaic Effect in Cadmium Sulfide. Phys. Rev. 1954, 96, 533-534. Bhattacharya, R. N.; Contreras, M. A.; Teeter, G. 18.5\% Copper Indium Gallium Diselenide (CIGS) Device Using SingleLayer, Chemical-Bath-Deposited ZnS(O,OH). Jpn. J. Appl. Phys. 2004, 43, L1475-L1476.

(46) Katagiri, H.; Jimbo, K.; Maw, W. S.; Oishi, K.; Yamazaki, M.; Araki, H.; Takeuchi, A. Development of CZTS-based thin film solar cells. Thin Solid Films 2009, 517, 2455-2460. 


\section{DISCLAIMER}

This document was prepared as an account of work sponsored by the United States Government. While this document is believed to contain correct information, neither the United States Government nor any agency thereof, nor the Regents of the University of California, nor any of their employees, makes any warranty, express or implied, or assumes any legal responsibility for the accuracy, completeness, or usefulness of any information, apparatus, product, or process disclosed, or represents that its use would not infringe privately owned rights. Reference herein to any specific commercial product, process, or service by its trade name, trademark, manufacturer, or otherwise, does not necessarily constitute or imply its endorsement, recommendation, or favoring by the United States Government or any agency thereof, or the Regents of the University of California. The views and opinions of authors expressed herein do not necessarily state or reflect those of the United States Government or any agency thereof or the Regents of the University of California. 